\title{
Phase referencing VLBI astrometry observation system: VERA
}

\author{
H. Kobayashi ${ }^{1}$, N. Kawaguchi ${ }^{1}$, S. Manabe ${ }^{1}$, K. M. Shibata ${ }^{1}$, \\ M. Honma ${ }^{1}$, Y. Tamura ${ }^{1}$ O. Kameya ${ }^{1}$, T. Hirota ${ }^{1}$, T. Jike ${ }^{1}$, H. Imai ${ }^{2}$, \\ and T. Omodaka ${ }^{2}$ \\ ${ }^{1}$ Mizusawa VERA Observatory, National Astronomical Observatory of Japan \\ ${ }^{2}$ Division of Astronomy, Department of Science, Kagoshima University \\ email: hideyuki.kobayashi@nao.ac.jp
}

\begin{abstract}
VERA aims at astrometric observations using phase referencing VLBI techniques, whose goal is a 10 micro arc-second accuracy for annual parallax measurements. VERA has four 20-m diameter VLBI radio telescopes in Japanese archipelago with the maximum baseline length of $2,300 \mathrm{~km}$. They have the two-beam observing system, which makes simultaneous observations of two objects possible. This leads to very accurate phase referencing VLBI observations. An important science goal is to make a 3-dimensional map of the Galaxy and reveal its dynamics. In order to achieve this, VERA has the $22 \mathrm{GHz}$ and $43 \mathrm{GHz}$ bands for $\mathrm{H} 2 \mathrm{O}$ and $\mathrm{SiO}$ maser objects, respectively. Maser objects are compact and suitable for astrometry observations. VERA's construction was started in 2000 and the array became operational in 2004. We have already measured annual parallaxes and proper motions of some galactic objects. In the future, VERA will collaborate with Korean and Chinese VLBI stations.
\end{abstract}

Keywords. instrumentation: interferometers, masers

\section{Introduction}

VLBI (Very Long Baseline Interferometer) technique provides the highest angular resolution in astronomical observation toolkit. Its typical angular resolution can be a few milli-arc-seconds or better. However, it was very difficult to determine the absolute position of the objects because of the uncertainty of absolute phases. Recently phase referencing technique has been developed, which usually makes observations using nodding two objects with a short time interval. However, phase errors still remain even after phasereferencing, which is caused by the short-term atmospheric fluctuations and instrumental instabilities. Thus, we have developed VERA (VLBI Exploration of Radio Astrometry) with 2-beam observing system, which allows us to perform simultaneous observation of two objects for accurate astrometric observations. The construction of VERA started in 2000 and completed in 2003. After the completion, verification and check-out were carried out for two years, the scientific observations started in 2004. In 2006, we have succeeded in measuring trigonometric parallaxes for some objects.

\section{System Overview of VERA}

VERA consists of four VLBI stations in Japanese archipelago (Fig. 1) with the maximum baseline length of 2,300 km and the minimum of 1,000 km. Observing frequency bands are 2, 8, 22, and $43 \mathrm{GHz}$. The 2 and $8 \mathrm{GHz}$ bands are used for geodesy observations and 22 and $43 \mathrm{GHz}$ bands are used for $\mathrm{H}_{2} \mathrm{O}$ and $\mathrm{SiO}$ maser observations, respectively. Each station has the same 20 -m diameter radio telescope with a 2-beam observational 


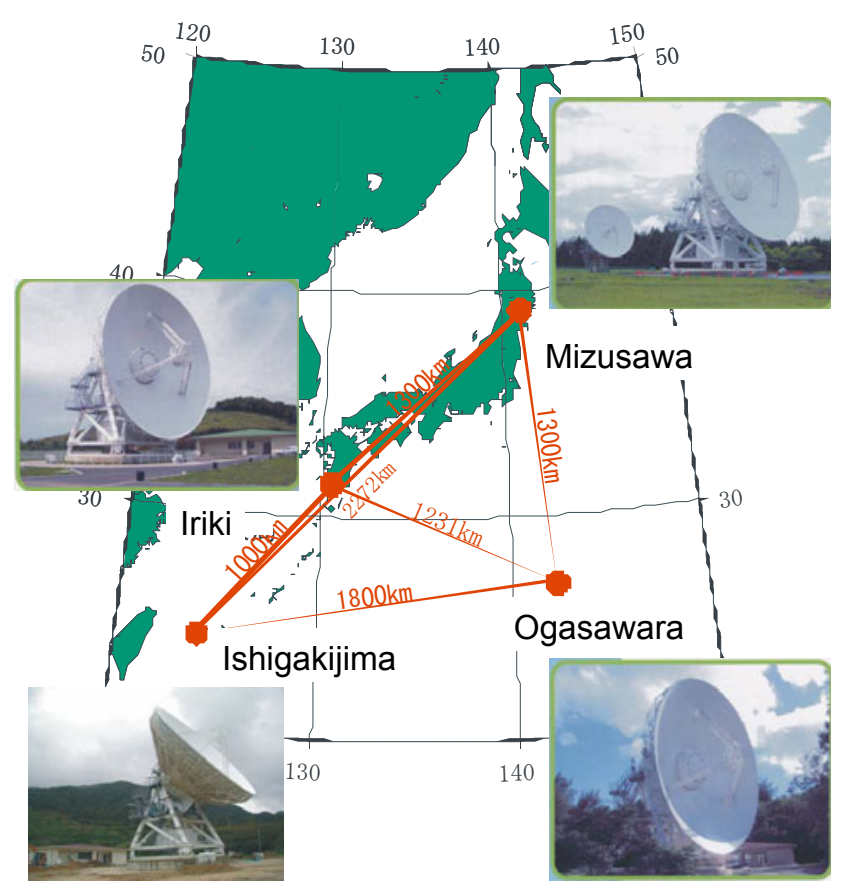

Figure 1. Distribution of VERA stations with baseline lengths of the array.

system. The 2-beam observational system makes it possible to observe two celestial objects simultaneously within a 2.2-deg separation angle. Usually one object is selected as a target source (e.g., a maser) and the other is an extra-galactic AGN object that serves as a phase calibrator. Observed phase variations of a calibrator is applied to the visibility data of the target object, and then a relative position of the target is determined with respect to the reference calibrator. Since the calibrator is extra-galactic and has no parallax, trigonometric parallax can be measured based on the relative astrometry between the target and the reference. In Fig. 2, a schematic view of the VERA 2-beam system is shown. Receivers can move over the focal plane of a telescope, which has a field curvature of its field-of-view at $2.2 \mathrm{deg}$.

In Fig. 3, an example of phase fluctuation measurements with two beams is shown (Honma et. al, 2003). In this observation, two strong maser sources were observed at the same time, and phase fluctuations of both sources showed a similar trend. In fact, the difference between the visibility phases is nearly flat, showing that phase-referencing with the 2-beam system works well to cancel out the fluctuations of troposphere.

For correlation process of the VERA array, we use tape-base recording system. The data are correlated with the Mitaka FX correlator. The correlator was originally developed for the VSOP (VLBI Space Observatory Program) project, and modified to VERA's high speed recording system, which provides a data rate of 1 Gpbs. The Mitaka FX correlator can process 5 stations at maximum data rate of 1 Gbps. 


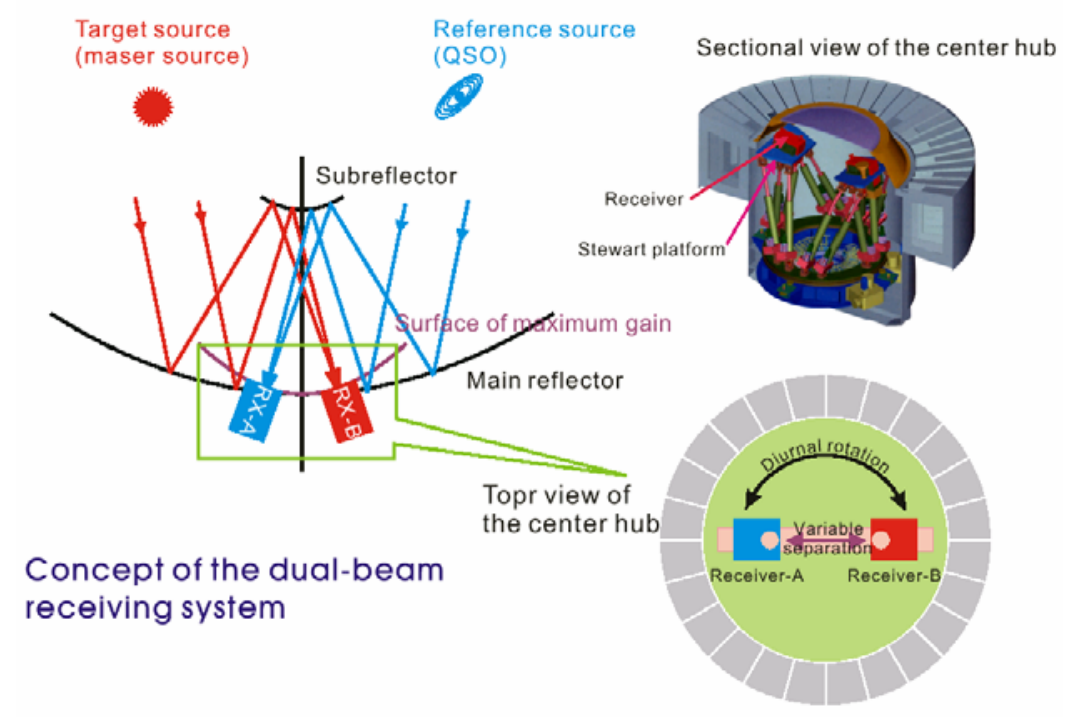

Figure 2. A schematic view of the 2-beam observing system with moving receivers.

\section{Scientific goal}

VERA is an astrometric tool for the galactic maser sources. $\mathrm{H}_{2} \mathrm{O}$ and $\mathrm{SiO}$ maser sources are thought to be compact and suitable for the astrometric observations. The scientific goal of VERA is the construction of the Galaxy's map based on trigonometric measurements of these masers. Also the dynamics of the Galaxy will be revealed by the measurements of the distances and proper motions. In order to make the Galaxy's map with an accuracy better than $20 \%$, one requires a parallax accuracy of 10 micro-arcsecond, which is the goal of VERA's measurement accuracy. Among thousands of known maser sources, we expect that we can measure the trigonometric parallax and proper motions of more than 500 sources. Possible distribution of candidate sources (based on estimated distances) is shown in Fig. 4. Since $\mathrm{H}_{2} \mathrm{O}$ and $\mathrm{SiO}$ maser sources are mostly starforming regions and late type stars (AGB stars), VERA will also provide new information for studies of these objects, in particular precise distances as well as internal gas motions near proto-stars as well as those in the circumstellar envelopes of AGB stars.

\section{Sensitivity}

The aperture efficiency of VERA $20-\mathrm{m}$ telescope is around $40 \%$ and $50 \%$ at $43 \mathrm{GHz}$ and $22 \mathrm{GHz}$, respectively. Typical system noise temperatures are $200 \mathrm{~K}$ and $400 \mathrm{~K}$ at 22 $\mathrm{GHz}$ and $43 \mathrm{GHz}$, respectively. Usual fringe detection sensitivity for AGN calibrators is $100 \mathrm{mJy}$ at $22 \mathrm{GHz}$, and is $250 \mathrm{mJy}$ at $43 \mathrm{GHz}$, both at $100 \mathrm{sec}$ of integration time. If the fringe of calibrators is detected, target sources are possible to integrate for a long time, e.g., 6 hours. Thus, the sensitivity of observations is limited by the flux intensity of a calibrator. In the case of a bright maser and a faint calibrator, one may use a maser source 


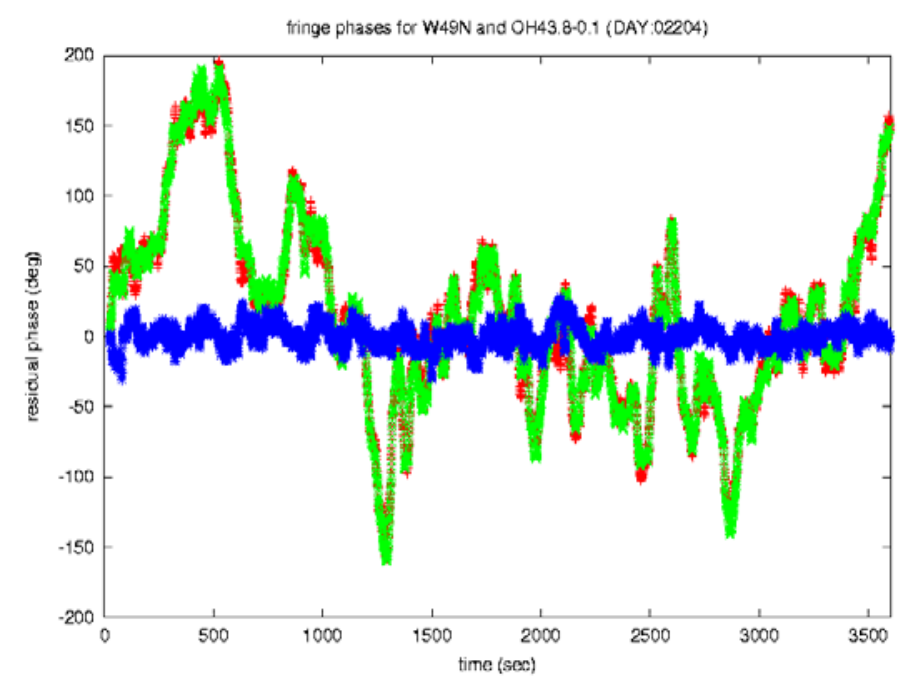

Figure 3. Phase variations of $\mathrm{W} 49 \mathrm{~N}$ and $\mathrm{OH} 43.8-0.1$ (rapidly-varying curves, nearly identical to each other), simultaneously observed with the 2-beam system. The difference of the two is also shown in the curve around 0 (Honma et al. 2003)

as a 'reference' to solve for the tropospheric fluctuations and obtain a phase-referenced map of that calibrator. This method can be used for masers with intensity of $\sim 10 \mathrm{Jy}$ or brighter. In both cases, a target and a reference should be located within 2.2 deg from each other, which is the mechanical limit of the 2-beam system.

\section{Geodesy}

The station position should be accurate for precise astrometric observations. For the astrometric accuracy of 10 micro-arc-second and a 2,300 km baseline length, $\sim 2 \mathrm{~mm}$ and $\sim 5 \mathrm{~mm}$ accuracies are required in horizontal and vertical directions, respectively. To achieve this, VERA performs VLBI geodetic observations every two weeks as well as continuous station-position measurements using GPS. VLBI observations for geodesy are carried out at $2 / 8 \mathrm{GHz}$ and also at $22 \mathrm{GHz}$. Currently the horizontal accuracy of $2 \mathrm{~mm}$ and vertical of $4 \mathrm{~mm}(1-\sigma)$ are achieved. Moreover, we plan to measure vertical displacements by using a gravity meter to improve the accuracy. Figure 5 shows comparison of baseline accuracies with various geodesy experiments. VERA has already achieved the top accuracy. 


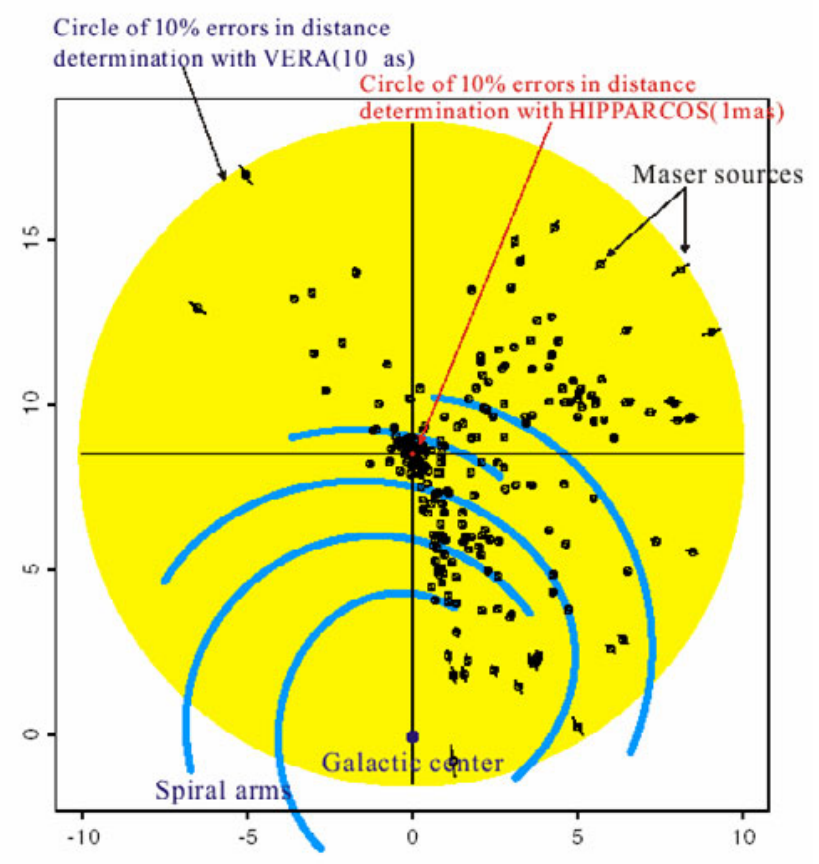

Figure 4. Possible distribution of $\mathrm{H}_{2} \mathrm{O}$ maser sources in the Galaxy. The filled circle corresponds to a area of $10 \%$ distance error with a parallax accuracy of $10 \mu$ as.

\section{Recent Results}

We have succeeded in measuring trigonometric parallax for some objects. For instance, toward Orion KL, which is one of the most famous massive star forming regions, we have measured the trigonometric parallax of $\mathrm{H}_{2} \mathrm{O}$ maser sources, providing the distance of $437 \pm 19$ pc (Hirota, et. al, 2007). Our distance is slightly smaller than the distance estimate of $480 \mathrm{pc}$ based on statistical parallax (Genzel et. al, 1981). Recently, parallax measurements of non-thermal stars in Orion region were published by other investigators, providing a distance of $414 \pm 7$ pc (Menten, 2007, Sandstrom, et. al, 2007). We are conducting astrometry of the $\mathrm{SiO}$ maser in Orion KL and its preliminary distance based on half a year worth of data is $419 \pm 6$ pc. When the observations of SiO maser are completed, it will hopefully provide the most accurate distance measurement to this Orion region.

In addition to Orion, around 20 sources are selected for regular astrometric monitoring to measure the distance and proper motions. For S269, we have successfully measured a parallax beyond $5 \mathrm{kpc}$ with its distance at $5.28 \pm 0.24 \mathrm{kpc}$ (Honma et al. 2007). S269 is one of the most distant sources for which a trigonometric parallax has been measured. Proper motions as well as parallax distance of S269 were used to constrain the Galactic rotation velocity at the position of S269, which is $13 \mathrm{kpc}$ away from the Galaxy center, providing a direct confirmation of flat rotation curve out to $13 \mathrm{kpc}$. For molecular cloud NGC 281, which is associated with an HI super-bubble and is located 300 pc away from the Galactic plane, Sato et al.(2007) found that the NGC 281 system has a systematic 


\section{Comparison of Baseline Standard Deviations}

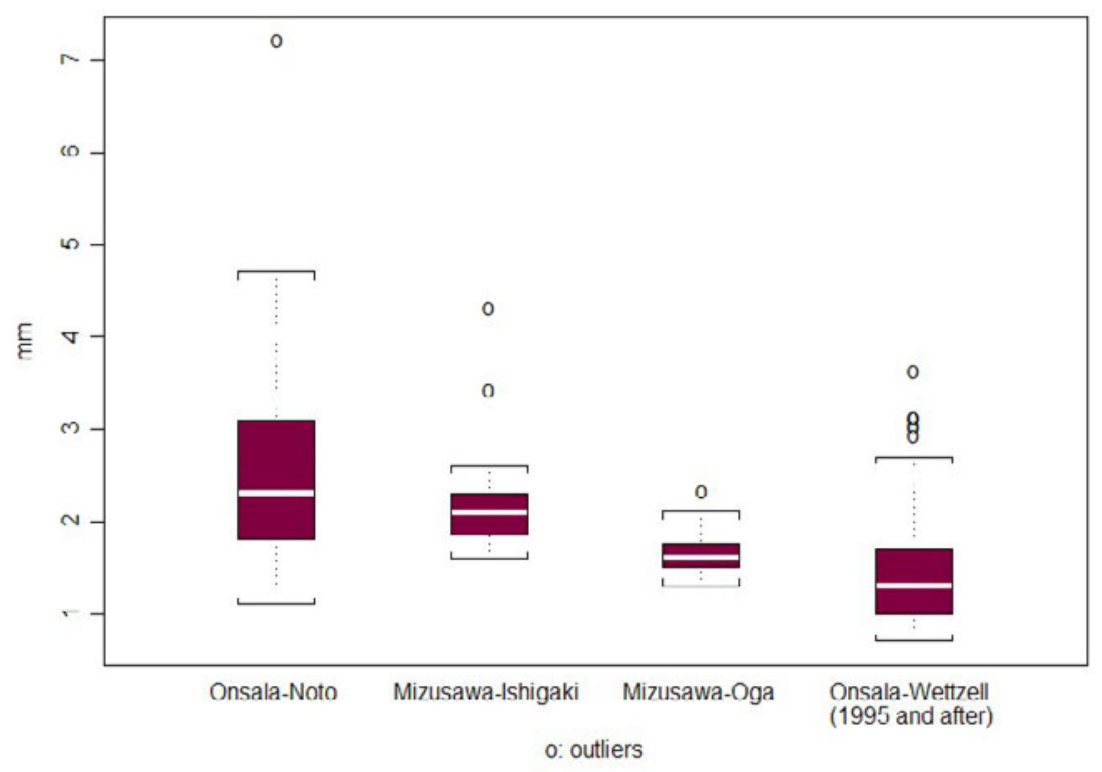

Figure 5. Comparison of baseline errors among geodesy experiments. VERA has achieved 2-3 $\mathrm{mm}$ accuracy in horizontal direction.

motion of $\sim 20 \mathrm{~km} / \mathrm{s}$, moving away from the Galactic plane. This is the first detection of proper motions for sources associated with Galactic super-bubble, and provides a direct evidence that these super-bubble were blown out from the Galaxy plane, most likely by multiple SNe (Sato et al. 2007).

Also, some of the nearby star-forming regions have been observed with VERA and the distances were already obtained for two molecular clouds. Hirota et al.(2008) obtained the parallax distance of NGC 1333 to be $235 \pm 18$ pc based on the $\mathrm{H}_{2} \mathrm{O}$ maser associated with SVS 13. The distance of $\rho$-Oph cloud was measured to be $178_{-37}^{+18}$ pc by Imai et al.(2007), which is consistent with the results recently obtained by Loinard et al. (2008) based on VLBA parallax measurements of radio-emitting young stars in this region. In addition to star forming regions, there are some late-type stars for which astrometric observations were successfully made. A distance measurement of nearby semi-regular variable star $\mathrm{S}$ Crt is reported in these proceeding (Nakagawa 2008), providing a parallax distance of $430 \pm 25$ pc. Choi et al.(2008) also measured a parallax distance of super-giant star VY CMa as $1.1 \pm 0.1 \mathrm{kpc}$, which is slightly smaller than the previous estimates.

\section{Future works}

Currently we have been carrying out 4 astrometric programs: 1) structure of nearby star-forming region, 2) galactic structure within $5 \mathrm{kpc}$ of the Sun, 3) period-luminosity relation for Mira variables and 4) distance measurement of the galactic center. More than 20 objects are programmed for the measurements of trigonometric parallaxes and 

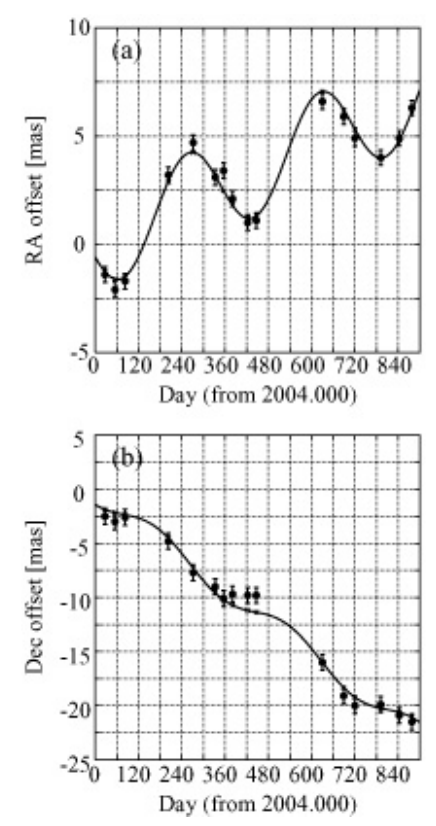

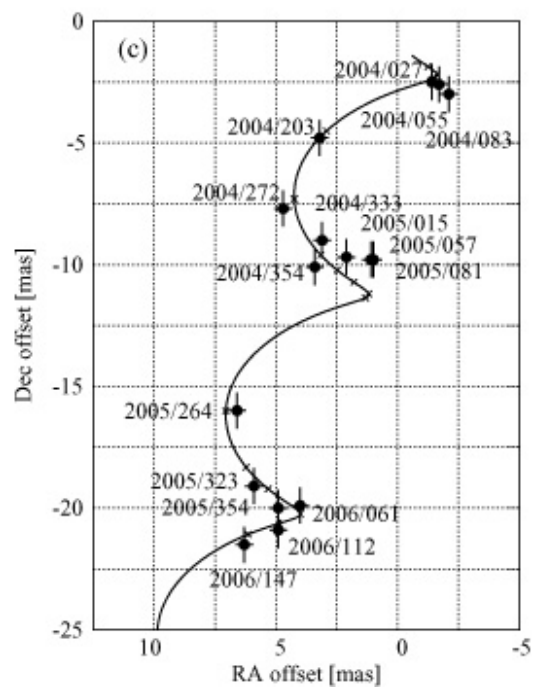

Figure 6. VERA measurement result for the trigonometric parallax and proper motions of Orion-KL $\mathrm{H}_{2} \mathrm{O}$ maser (Hirota et al. 2007).

proper motions. In addition to an operation as VERA, we have a plan to collaborate with Korean VLBI Network (KVN), which will have multi-frequency phase referencing capability. KVN will have 3 telescopes in Korea with 21-m diameter, with operating frequency up to $129 \mathrm{GHz}$. The combined Korean-Japanese VLBI array will have a dense UV coverage, providing shorter baselines than those of VERA itself and, hence, yielding a better imaging. Also, China has a 4-station VLBI network, called the CVN (Chinese VLBI Network). We have started discussions about an East Asia VLBI network which would include China, Japan, and Korea. Figure 7 shows the distribution of VLBI stations in this area. Including VLBI stations operated by Japanese universities and research intitutes other than NAOJ, there will be nearly 20 VLBI stations in the East Asian region. Thus, when these stations are combined to form a bigger array, the array will provide a better imaging capability and a higher resolution, and thus will significantly contribute to the better accuracy in VLBI astrometry. 


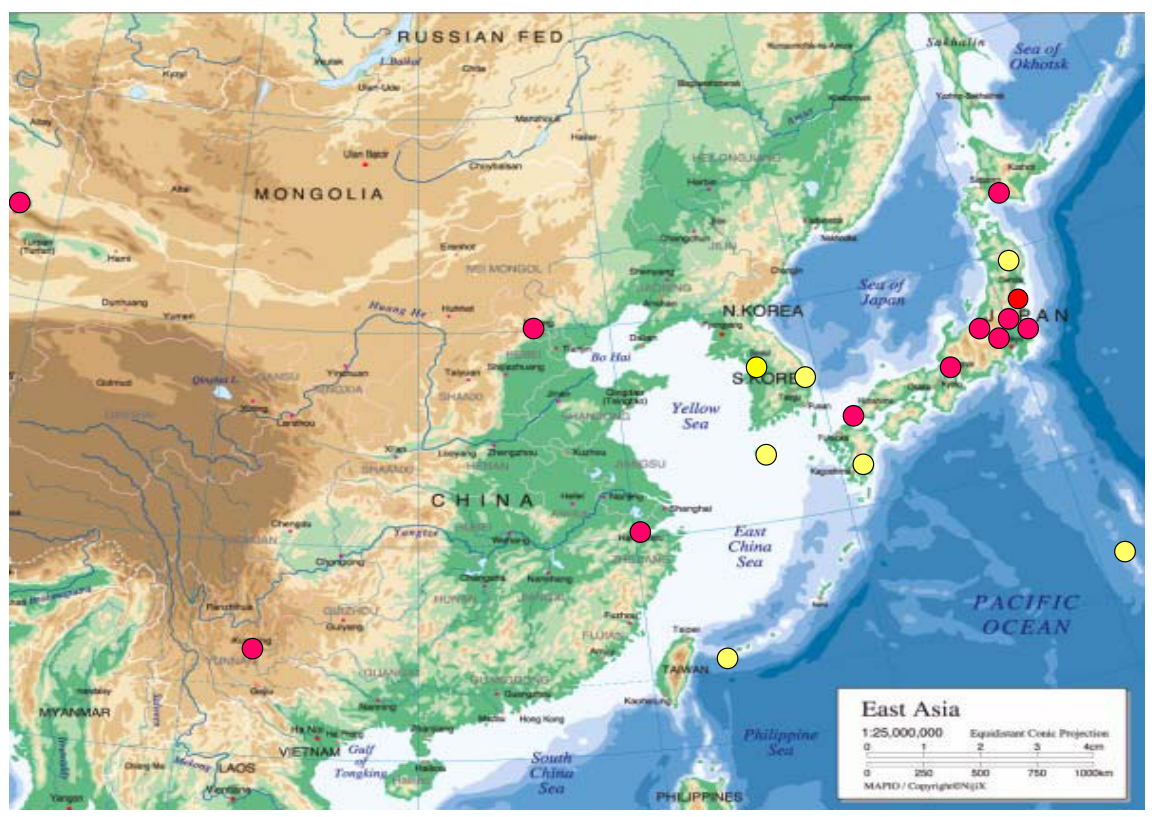

Figure 7. Distribution of VLBI stations in the East Asia region. The East Asia VLBI Network is under consideration.

\section{References}

Choi, Y. K., et al., 2008, in this volume p.192

Genzel, R., et al., 1981, ApJ, 244, 884

Loinard, L., et al., 2008, in this volume p.186

Hirota, T., et al., 2007, PASJ, 59, 897

Honma, M., et al., 2003, PASJ, 55, L57

Honma, M., et al., 2007, PASJ, 59, 889

Menten, K., et al., 2007, A\&SA, 474, 515

Nakagawa, A., et al., 2008, this volume p.206

Sandstrom, M., et al., 2007, ApJ, 667, 1161

Sato, M., et al., 2007, PASJ, 59, 743 